\title{
STATISTICAL OPTIMIZATION OF MEDIUM COMPONENTS BY PLACKETT BURMAN DESIGN AND RESPONSE SURFACE METHODOLOGY FOR ENHANCED ANTIOXIDANT ACTIVITY BY XYLARIA FEEJEENSIS HMJAU22039
}

\author{
DURGA REBBAPRAGADA*, RAJAGOPAL KALYANARAMAN \\ Department of Biotechnology, School of Life Sciences, Vels University, Pallavaram, Chennai, India \\ Email: pallavi_durga@yahoo.co.in
}

Received: 22 Jul 2016 Revised and Accepted: 09 Sep 2016

\begin{abstract}
Objective: To enhance the antioxidant potential of Xylaria feejeensis by statistical optimization. The components of potato dextrose yeast extract (PDYEB) medium: potato infusion, dextrose and yeast extract were investigated to optimize antioxidant activity by Plackett-Burman design (P-B design). The interaction between the critical components of the medium and incubation time was further investigated by Response Surface Method (RSM) and the culture conditions were optimized for enhancing antioxidant activity. Central composite design (CCD) was employed and quadratic response surface model was used to analyze the data using analysis of variance (ANOVA).
\end{abstract}

Methods: The antioxidant potential was measured by 1-1diphenyl-2-picryl-hydrazil (DPPH) radical scavenging assay, nitric oxide (NO) scavenging assay. The antioxidant activity was compared with total phenolic content measured by Folin-Ciocalteau (FC) reagent based assay.

Results: Plackett-Burman design revealed dextrose and yeast extract to be the most signficant components of PDYEB medium ( $<$.0001). ANOVA analysis showed that the model was highly significant $(\mathrm{p}<0.0001)$ for antioxidant activity measured by DPPH, NO scavenging and TPC (total phenolic content). The model was justified by applying the optimized conditions and values of $87.38 \%, 56.43 \%, 27.28 \mathrm{mg} / \mathrm{g}$ for DPPH, NO scavenging and TPC respectively were obtained. The estimated optimum conditions of the variables for the antioxidant activity and phenolic content are $12.72 \mathrm{~g}$ of dextrose, $1.67 \mathrm{~g}$ yeast extract, and incubation period of $23.43 \mathrm{~d}$.

Conclusion: The study highlights the importance of various components of the medium and the physiochemical limitations in antioxidant potential and phenol production of the fungal strain.

Keywords: DPPH, TPC, Endophytic fungi, Central composite design

(C) 2016 The Authors. Published by Innovare Academic Sciences Pvt Ltd. This is an open access article under the CC BY license (http://creativecommons.org/licenses/by/4. 0/) DOI: http://dx.doi.org/10.22159/ijpps.2016v8i11.14257

\section{INTRODUCTION}

Antioxidants neutralize free radicals produced during physiological processes that cause aging, inflammation, and neurodegenerative disorders [1]. Synthetic antioxidants are reported to be carcinogenic [2] so antioxidant properties of products from natural resources are characterized, isolated and identified. Recent investigations reported that the extracts of Xylaria feejeensis have high antioxidant activity. Physicochemical conditions of the culture considerably influence the growth and metabolite production of fungal strain [3]. One-factor-at-atime approach of optimization ignores the true optimum conditions and the interaction effect of the different variables in the medium. A number of statistical designs are used to search the key factors of a medium rapidly from a multivariable system. Plackett-Burman design and response surface methodology are prominent statistical optimization techniques that allow quick screening of critical components of media and their interactive effects.

In a broader investigation which included a study on the diversity of endophytic fungi from Tectona grandis Linn. f the seasonal endophytic assemblages that were isolated were screened for antioxidant potential. A total of 14 strains were isolated of which Xylaria feejeensis reported highest antioxidant activity during preliminary screening by DPPH. Hence in the present study, the antioxidant potential of endophytic fungal isolate Xylaria feejeensis was measured by DPPH assay and NO scavenging assay using Plackett-Burman design and Central composite design of RSM. The total phenolic content of the extract was also recorded and the correlation between phenolic content and antioxidant activity was noted. The Plackett-Burman design is a widely used statistical technique for screening and selection of most important culture variables from a multivariable medium [4]. This design is a tool for preliminary optimization and evaluation of the importance of different medium components. This design evaluates two levels of the medium components and their influence on the activity. It does not provide for the optimal quantity of the components required in the medium. RSM which is an important tool in medium optimization employs mathematical and statistical techniques in designing experiments and evaluating the effect of variables [5]. The objective of this study was to generate a model to optimize and enhance the antioxidant potential of the fungal strain. It involves an experimental plan such as Central Composite Design to fit a second-order polynomial by least squares method. For 1 numeric variable, CCD has 5 levels $f \alpha,-1,0,+1,+\alpha)$. An equation is used to describe the test variables, and the combined and interactive effect of all the variables in the response. RSM also predicts the interaction and correlation between the experimental variables and observed results and subsequently provide optimized conditions.

\section{MATERIALS AND METHODS}

Isolation and identification

The endophyte Xylaria feejeensis HMJAU22039 was isolated from Tectona grandis Linn. $f$. Healthy leaf segments of Tectona grandis were surface sterilized and inoculated on potato dextrose agar (PDA) medium. After $1 \mathrm{w}$ of growth, the fungal strain was identified by morphological features and the culture was transferred to PDA slants and stored at $4^{\circ} \mathrm{C}$. Molecular identification by ITS region of rRNA of the strain was confirmed by NFCCI, Pune. All the chemicals and media/components were purchased from Hi-Media Pvt. Ltd., Mumbai, India. All the solvents used in the study were of analytical grade.

\section{Antioxidant activity assays}

Free-radical-scavenging activity measured by 1-1diphenyl-2picryl-hydrazil (DPPH)

The free radical scavenging activity of the ethyl acetate extracts was measured by DPPH radical scavenging assay [6]. An aliquot of $1 \mathrm{ml}$ 
of $0.1 \mathrm{mmol}$ DPPH solution in methanol and $0.5 \mathrm{ml}$ of extract were mixed, shaken vigorously and incubated at room temperature for 30 min. The absorbance was measured at $517 \mathrm{~nm}$. The DPPH radical scavenging effect was calculated according to the equation:

$$
\text { DPPH scavenging efffect }(\%)=\frac{(\mathrm{A} 0-\mathrm{A} 1)}{(\mathrm{A} 0)} \times 100
$$

Where A0 is the absorbance of the control and A1 is the absorbance of the sample.

\section{Determination of nitric oxide (NO) scavenging activity}

$2 \mathrm{ml}$ of sodium nitroprusside ( $5 \mathrm{mmol}$ ) solution was mixed with $2 \mathrm{ml}$ of extract and incubated at room temperature for two and half hours. $0.5 \mathrm{ml}$ of the reaction mixture was mixed with an equal amount of Griess reagent. The absorbance was measured at $546 \mathrm{~nm}$ [7]. The NO scavenging effect was calculated according to the equation:

$$
\text { NO scavenging efffect }(\%)=\frac{(\mathrm{A} 0-\mathrm{A} 1)}{(\mathrm{A} 0)} \times 100
$$

Where A0 is the absorbance of the control reaction and A1 is the absorbance of the sample.

\section{Determination of total phenolic content (TPC)}

Total phenol content was estimated using Folin-Ciocalteau (FC) reagent based assay [8]. The extract was dissolved in methanol $(1 \mathrm{mg} / \mathrm{ml})$ and $500 \mu \mathrm{l}$ of $(50 \%) \mathrm{FC}$ reagent was added followed by the addition of $1.5 \mathrm{ml}$ of $20 \%$ of $\mathrm{Na}_{2} \mathrm{CO}_{3}$. The final volume was made up to $5 \mathrm{ml}$ by adding distilled water. The mixture was kept at $28 \mathrm{C}$ for $30 \mathrm{~min}$ and the absorbance was recorded at $765 \mathrm{~nm}$. This process was repeated to 1 to of $10-100 \mu \mathrm{g} / \mathrm{ml}$ methanolic gallic acid solutions which were used as a standard. Total phenolic values of the samples were obtained from the regression equation: $\mathrm{y}=0.006141 \mathrm{x}+0.157733$ with $\mathrm{R}^{2}=0.9975$ and expressed as $\mathrm{mg} / \mathrm{g}$ gallic acid equivalents.

\section{Statistical optimization of the medium}

Plackett-burman (P-B) design for analyzing significant components of the medium

After a preliminary study on different media, potato dextrose yeast extract medium (PDYEB) was selected for PB design with an aim to identify the significant components of the medium that have a critical role in antioxidant potential of the endophytic fungus Xylaria feejeensis. The 3 factors, which are components of PDYEB medium (potato infusion, dextrose, yeast extract) were examined. Total 10 tests were designed including 8 combinations and 2 repetitions at a central point which contain a different concentration of each factor (table 1).

Lower and upper levels of the variables are represented by-1 and +1 levels. To determine the effect of each factor the difference between the responses (+and-) were considered. The statistical significance of the responses was ascertained using students T-test and p-value. The larger the magnitude of T-value and smaller $\mathrm{P}$ value, the more significant is the corresponding coefficient. All the experiments were performed in $500 \mathrm{ml}$ Erlenmeyer flask containing $250 \mathrm{ml}$ of media.

Table 1: Variables of PDYEB medium and their values for P-B design

\begin{tabular}{llll}
\hline Variable & Units & $\mathbf{- 1}$ & $\mathbf{1}$ \\
\hline Potato infusion & $\mathrm{g}$ & 100 & 300 \\
Dextrose & $\mathrm{g}$ & 10 & 30 \\
Yeast extract & $\mathrm{g}$ & 1 & 3 \\
\hline
\end{tabular}

\section{Response surface method (RSM) of medium optimization using central composite design (CCD)}

Design expert version 10 statistical software was used to obtain optimal working conditions for the experiment, generate response surface graphs and perform statistical analysis of data. Central composite design of RSM was used for the experiment and dextrose, yeast extract and incubation period were taken as independent variables for the optimization of antioxidant activity. The 5 levels at which dextrose, yeast extract and incubation period were studied are $(3.18,10,20,30,36.81) \mathrm{g},(0.31,1,2,3,3.68) \mathrm{g}$, and $(11.59,15$, $20,25,28.40$ ) d respectively (table 2 ).

The experimental design included 20 flasks with six replicates having all the three variables at their central coded values. All the experiments were performed in $500 \mathrm{ml}$ Erlenmeyer flask containing $250 \mathrm{ml}$ of media. DPPH radical scavenging assay, NO scavenging assay and total phenolic content were taken as responses $\mathrm{P}_{(1-3)}$. The mathematical relationship of response $\mathrm{P}$ (for each parameter) and independent variable $\mathrm{X}\left(\mathrm{X}_{\mathrm{A}}\right.$ : dextrose; $\mathrm{X}_{\mathrm{B}}$ : yeast extract; $\mathrm{X}_{\mathrm{C}}$ : incubation period) was calculated by the following quadratic model equation:

$$
\begin{aligned}
P_{(1,2,3)}=\beta_{0}+\beta_{A} X_{A}+ & \beta_{B} X_{B}+\beta_{C} X_{C}+\beta_{A A} X_{A}^{2}+\beta_{B B} X_{B}^{2}+\beta_{C C} X_{C}^{2}+\beta_{A B} X_{A} X_{B} \\
& +\beta_{A C} X_{A} X_{C}+\beta_{B C} X_{B} X_{C}
\end{aligned}
$$

Where $\mathrm{P}$ is the predicted response for the parameter; $\beta_{0}$ : intercept; $\beta_{A}, \beta_{B}$, and $\beta_{C}$ : linear coefficients; $\beta_{A A}, \beta_{B B}$ and $\beta_{C C}$ : squared coefficients $\beta_{\mathrm{AB}}, \beta_{\mathrm{AC}}$, and $\beta_{\mathrm{BC}}$ : interaction coefficients.

\section{RESULTS}

\section{Plackett-burman design for selection of significant components}

Antioxidant potential of Xylaria feejeensis assayed by DPPH and NO radical scavenging assay and total phenolic content varied significantly with the 10 run of different combinations of the media components (potato infusion, dextrose, yeast extract) evaluated by the Plackett-Burman design of experiments. The maximum antioxidant potential along with high TPC was observed in run order 5 and run order 8 which was followed by run order 6 (table 3).

The results were subjected to regression analysis and the analysis of variance (ANOVA) which revealed dextrose to have statistically significant effect on antioxidant potential with $P$ value $\leq 0.01$ for all three responses. The $\mathrm{P}$ value of yeast extract was also statistically significant with $P$ value $\leq 0.01$ for DPPH and NO and $p \leq 0.05$ for TPC. The $P$ value of potato infusion was statistically not significant with $P$ value $0.72,0.63$ and 0.84 for DPPH, NO and TPC respectively (table 4). This showed that dextrose and yeast extract played a critical role for antioxidant activity.

Table 2: Variables for CCD of RSM and their values

\begin{tabular}{llllll}
\hline Variable & Units & $-\boldsymbol{\alpha}$ & $\mathbf{- 1}$ & $\mathbf{0}$ & $\mathbf{1}$ \\
\hline Dextrose & $\mathrm{g}$ & 3.18207 & 10 & 20 & 30 \\
Yeast extract & $\mathrm{g}$ & 0.318207 & 1 & 2 & 3 \\
Incubation time & $\mathrm{d}$ & 11.591 & 15 & 3.68179 & 20 \\
\hline
\end{tabular}


Table 3: Variables of PDYEB medium and their values for P-B design

\begin{tabular}{|c|c|c|c|c|c|c|}
\hline \multirow[t]{2}{*}{ Run order } & \multicolumn{3}{|l|}{ Variables } & \multicolumn{3}{|c|}{ Antioxidant activity } \\
\hline & Potato infusion (g) & Dextrose (g) & Yeast extract (g) & DPPH (\%) & NO scavenging (\%) & TPC (mg/g) \\
\hline 1 & 100 & 10 & 1 & $30.75 \pm 0.34$ & $21.01 \pm 0.44$ & $8.67 \pm 0.1$ \\
\hline 2 & 100 & 30 & 1 & $49.23 \pm 0.48$ & $32.17 \pm 0.57$ & $16.82 \pm 0.29$ \\
\hline 3 & 300 & 30 & 1 & $49.81 \pm 0.55$ & $32.39 \pm 0.22$ & $17.02 \pm 0.56$ \\
\hline 4 & 300 & 10 & 3 & $38.47 \pm 0.31$ & $26.34 \pm 0.28$ & $12.37 \pm 0.36$ \\
\hline 5 & 200 & 20 & 2 & $72.49 \pm 0.37$ & $48.95 \pm 0.53$ & $22.81 \pm 0.18$ \\
\hline 6 & 300 & 30 & 3 & $61.06 \pm 0.25$ & $41.04 \pm 0.47$ & $19.11 \pm 0.3$ \\
\hline 7 & 100 & 30 & 3 & $60.23 \pm 0.42$ & $40.19 \pm 0.24$ & $18.89 \pm 0.38$ \\
\hline 8 & 200 & 20 & 2 & $71.98 \pm 0.14$ & $48.12 \pm 0.52$ & $22.2 \pm 0.15$ \\
\hline 9 & 100 & 10 & 3 & $38.98 \pm 0.5$ & $26.43 \pm 0.23$ & $12.98 \pm 0.11$ \\
\hline 10 & 300 & 10 & 1 & $31.02 \pm 0.45$ & $21.59 \pm 0.17$ & $9.23 \pm 0.2$ \\
\hline
\end{tabular}

DPPH: DPPH radical scavenging activity; NO: NO scavenging activity; TPC: total phenolic content. All values are reported as mean \pm SD ( $\mathrm{n}=3$ ).

Table 4: $P$ and $T$ values of components of $P-B$ design and their effect

\begin{tabular}{|c|c|c|c|c|c|c|c|c|c|}
\hline \multirow[t]{2}{*}{ Term } & \multicolumn{3}{|l|}{ DPPH } & \multicolumn{3}{|l|}{ NO } & \multicolumn{3}{|l|}{ TPC } \\
\hline & Effect & T Value & P Value & Effect & T Value & P Value & Effect & T Value & P Value \\
\hline Potato infusion & 0.292 & 0.376 & 0.721 & 0.39 & 0.508 & 0.632 & 0.092 & 0.211 & 0.840 \\
\hline Dextrose & 20.27 & 26.10 & $<0.001$ & 12.605 & 16.43 & $<0.001$ & 7.147 & 16.35 & $<0.001$ \\
\hline Yeast extract & 9.482 & 12.20 & $<0.001$ & 6.71 & 8.751 & $<0.001$ & 2.902 & 6.643 & $<0.005$ \\
\hline
\end{tabular}

DPPH: DPPH radical scavenging activity; NO: NO scavenging activity; TPC: total phenolic content.

\section{Central composite design}

Based on the results from P-B design, dextrose and yeast extract were selected as two important variables of PDYEB medium and CCD (RSM) was applied to optimize the conditions. To know the optimum incubation period and its interaction with dextrose and yeast extract, it was chosen as a third variable as it is the important physical parameter that affects the antioxidant activity. Antioxidant potential of Xylaria feejeensis assayed by DPPH and NO radical scavenging assay and total phenolic content varied significantly with the 20 run of different combinations of CCD design. Table 5 shows variables and their levels for CCD.

Table 5: Variables of PDYEB medium and their values for P-B design

\begin{tabular}{|c|c|c|c|c|c|c|}
\hline \multirow[t]{3}{*}{ Run } & \multicolumn{3}{|l|}{ Variables } & \multicolumn{3}{|c|}{ Antioxidant activity } \\
\hline & Dextrose & Yeast extract & Incubation time & DPPH & NO scavenging & TPC \\
\hline & g & g & d & $\%$ & $\%$ & $\mathrm{mg} / \mathrm{g}$ \\
\hline 1 & 10 & 1 & 25 & $88.45 \pm 0.56$ & $58.28 \pm 0.45$ & $27.48 \pm 0.11$ \\
\hline 2 & 10 & 3 & 25 & $81.79 \pm 0.44$ & $51.36 \pm 0.57$ & $23.11 \pm 0.18$ \\
\hline 3 & 30 & 3 & 15 & $75.12 \pm 0.41$ & $45.89 \pm 0.33$ & $18.34 \pm 0.37$ \\
\hline 4 & 30 & 3 & 25 & $52.65 \pm 0.35$ & $25.6 \pm 0.46$ & $8.41 \pm 0.1$ \\
\hline 5 & 10 & 1 & 15 & $59.82 \pm 0.39$ & $33.34 \pm 0.32$ & $12.17 \pm 0.31$ \\
\hline 6 & 10 & 3 & 15 & $59.59 \pm 0.3$ & $33.96 \pm 0.47$ & $11.84 \pm 0.33$ \\
\hline 7 & 30 & 1 & 25 & $63.67 \pm 0.27$ & $35.48 \pm 0.17$ & $13.75 \pm 0.11$ \\
\hline 8 & 20 & 2 & 20 & $87.32 \pm 0.52$ & $58.64 \pm 0.4$ & $28.43 \pm 0.39$ \\
\hline 9 & 20 & 2 & 20 & $86.44 \pm 0.36$ & $56.53 \pm 0.29$ & $27.54 \pm 0.13$ \\
\hline 10 & 20 & 2 & 20 & $88.13 \pm 0.22$ & $58.94 \pm 0.26$ & $28.58 \pm 0.36$ \\
\hline 11 & 30 & 1 & 15 & $80.15 \pm 0.58$ & $50.27 \pm 0.16$ & $22.12 \pm 0.15$ \\
\hline 12 & 20 & 2 & 20 & $87.65 \pm 0.48$ & $57.32 \pm 0.5$ & $27.62 \pm 0.1$ \\
\hline 13 & 3.18207 & 2 & 20 & $65.57 \pm 0.14$ & $36.18 \pm 0.18$ & $15.21 \pm 0.28$ \\
\hline 14 & 20 & 2 & 20 & $81.51 \pm 0.23$ & $46.84 \pm 0.49$ & $23.37 \pm 0.3$ \\
\hline 15 & 20 & 2 & 28.409 & $62.99 \pm 0.59$ & $34.03 \pm 0.23$ & $13.32 \pm 0.2$ \\
\hline 16 & 36.8179 & 2 & 20 & $57.95 \pm 0.34$ & $30.28 \pm 0.25$ & $11.22 \pm 0.21$ \\
\hline 17 & 20 & 3.68179 & 20 & $62.75 \pm 0.24$ & $34.68 \pm 0.55$ & $13.22 \pm 0.14$ \\
\hline 18 & 20 & 2 & 11.591 & $57.57 \pm 0.54$ & $30.94 \pm 0.57$ & $9.01 \pm 0.12$ \\
\hline 19 & 20 & 2 & 20 & $80.84 \pm 0.19$ & $50.95 \pm 0.25$ & $22.7 \pm 0.28$ \\
\hline 20 & 20 & 0.318207 & 20 & $72.27 \pm 0.38$ & $42.71 \pm 0.43$ & $17.02 \pm 0.42$ \\
\hline
\end{tabular}

DPPH: DPPH radical scavenging activity; NO: NO scavenging activity; TPC: total phenolic content. All values are reported as mean \pm SD ( $\mathrm{n}=3$ ).

\section{Significance of model}

Based on the sequential model sum of squares response surface, the Quadratic model is selected as it is the highest order polynomial, where the additional terms are significant, and the model is not aliased. The model F-value of 529.18, 125.36, 217 for DPPH, NO scavenging and TPC respectively implies the model is significant (table 6). There is only a $0.01 \%$ chance that an F-value this large could occur due to noise. The lack of fit P-value of 1.44, 0.22, and 1.93 for DPPH, $\mathrm{NO}$ and TPC implies the lack of fit is not significant relative to the pure error. Non-significant lack of fit is good for the model to fit. The predicted R-squared for all the three responses is in reasonable agreement with the adjusted R-squared i.e. the difference is less than 0.2 . Adequate precision measures the signal to noise ratio. A ratio greater than 4 is desirable for a good model. The ratios for DPPH, NO and TPC, are 65.504, 32.581, 40.48 indicate an adequate signal.

\section{Effect of different variables}

Dextrose, yeast extract and incubation time signi ficantly affected the DPPH activity. The linear effect $(A, B, C)$, squared effect $\left(A^{2}, B^{2}, C^{2}\right)$ as well as interactive effect $(A B, A C, B C)$ were highly sigirant $(P$ value $<0.01)$. For NO scavenging activity linear effect $(A, B)$, squared effect $\left(A^{2}, B^{2}, C^{2}\right)$ and interactive effect $(A C, B C)$ was highly 
significant with $P$ value $\leq 0.01$. While linear(C) was significant with $P$ value $\leq 0.05$ and interactive effect $(A B)$ showed less significance at $P$ $=0.062$. For total phenolic content the linear effect $(A, B, C)$, squared effect $\left(A^{2}, B^{2}, C^{2}\right)$ and interactive effect $(A C)$ was highly sigficant with $P$ value 0.01 . While interactive e ffect $(A B, B C)$ showed significance at $P$ value $\leq 0.05$ (table 7)

Table 6: Variables of PDYEB medium and their values for P-B design

\begin{tabular}{lll}
\hline & DPPH (\%) & NO scavenging (\%) \\
\hline Model (F value) & 529.18 & 125.36 \\
Lack of fit (F value) & 1.44 & 0.22 \\
Degrees of freedom & 19 & 19 \\
R-Squared & 0.9981 & 0.9921 \\
Adjusted R-Squared & 0.9962 & 0.9842 \\
Predicted R-Squared & 0.9937 & 0.9747 \\
Adequate Precision & 65.504 & 32.581 \\
\hline
\end{tabular}

Table 7: Variables of PDYEB medium and their values for P-B design

\begin{tabular}{|c|c|c|c|c|c|c|c|c|c|c|}
\hline & I & A & B & $\mathrm{C}$ & AB & AC & BC & $\mathbf{A}^{2}$ & $\mathbf{B}^{2}$ & $\mathrm{C}^{2}$ \\
\hline DPPH & 84.04 & -2.26 & -2.85 & 1.53 & -1.14 & -11.2 & -1.55 & -6.45 & -4.41 & -6.97 \\
\hline$P$ value & & $<0.01$ & $<0.01$ & $<0.01$ & $<0.01$ & $<0.01$ & $<0.01$ & $<0.01$ & $<0.01$ & $<0.01$ \\
\hline NO & 53.51 & -2.16 & -2.49 & 0.91 & -0.99 & -9.67 & -1.63 & -5.79 & -3.85 & -6.05 \\
\hline$P$ value & & $<0.01$ & $<0.01$ & $<0.05$ & $<0.1$ & $<0.01$ & $<0.01$ & $<0.01$ & $<0.01$ & $<0.01$ \\
\hline TPC & 25.59 & -1.36 & -1.47 & 1.13 & -0.55 & -5.61 & -0.7 & -3.56 & -2.89 & -4.29 \\
\hline$P$ value & & $<0.01$ & $<0.01$ & $<0.01$ & $<0.05$ & $<0.01$ & $<0.05$ & $<0.01$ & $<0.01$ & $<0.01$ \\
\hline
\end{tabular}

I: Intercept; A: Dextrose; B: Yeast extract; C: Incubation time

The data obtained from the quadratic model equation was found to be signi ficant. The $\mathrm{F}$ value and $\mathrm{R}^{2}$ value showed that the model correlated well with measured data and was statistically significant. The final predictive equations for each response: DPPH assay $\left(\mathrm{P}_{1}\right)$, NO scavenging assay $\left(\mathrm{P}_{2}\right)$, Total phenolic content $\left(\mathrm{P}_{3}\right)$, obtained are as follow:

Final equation in terms of coded factors:

$$
\begin{gathered}
\mathrm{P}_{1}=84.05-2.26 \mathrm{X}_{\mathrm{A}}-2.85 \mathrm{X}_{\mathrm{B}}+1.54 \mathrm{X}_{\mathrm{C}}-1.15 \mathrm{X}_{\mathrm{A}} \mathrm{X}_{\mathrm{B}}-11.22 \mathrm{X}_{\mathrm{A}} \mathrm{X}_{\mathrm{C}}-1.55 \mathrm{X}_{\mathrm{B}} \mathrm{X}_{\mathrm{C}} \\
-6.45 \mathrm{X}_{\mathrm{A}}^{2}-4.42 \mathrm{X}_{\mathrm{B}}^{2}-6.98 \mathrm{X}_{\mathrm{C}}^{2} \\
\mathrm{P}_{2}=53.52-2.17 \mathrm{X}_{\mathrm{A}}-2.49 \mathrm{X}_{\mathrm{B}}+0.91 \mathrm{X}_{\mathrm{C}}-1 \mathrm{X}_{\mathrm{A}} \mathrm{X}_{\mathrm{B}}-9.68 \mathrm{X}_{\mathrm{A}} \mathrm{X}_{\mathrm{C}}-1.63 \mathrm{X}_{\mathrm{B}} \mathrm{X}_{\mathrm{C}} \\
- \\
-5.79 \mathrm{X}_{\mathrm{A}}^{2}-3.86 \mathrm{X}_{\mathrm{B}}^{2}-6.05 \mathrm{X}_{\mathrm{C}}^{2} \\
\mathrm{P}_{3}=25.59-1.37 \mathrm{X}_{\mathrm{A}}-1.48 \mathrm{X}_{\mathrm{B}}+1.14 \mathrm{X}_{\mathrm{C}}-0.55 \mathrm{X}_{\mathrm{A}} \mathrm{X}_{\mathrm{B}}-5.61 \mathrm{X}_{\mathrm{A}} \mathrm{X}_{\mathrm{C}}-0.7 \mathrm{X}_{\mathrm{B}} \mathrm{X}_{\mathrm{C}} \\
-3.57 \mathrm{X}_{\mathrm{A}}^{2}-2.89 \mathrm{X}_{\mathrm{B}}^{2}-4.29 \mathrm{X}_{\mathrm{C}}^{2}
\end{gathered}
$$

In the response surface contour plot (DPPH A, TPC A), incubation time is held at a $20 \mathrm{~d}$ and levels of glucose and yeast extract are varied from- 1 to +1 . In the response surface contour plot (DPPH B,
TPC B), yeast extract is held at $2 \mathrm{~g} / \mathrm{l}$ and levels of dextrose and incubation time are varied from- 1 to +1 . In the response surface contour plot (DPPH C, TPC C), dextrose is held at $20 \mathrm{~g} / \mathrm{l}$ and levels of incubation time and yeast extract are varied from-1 to+1 (fig. 1). The corresponding contour plots indicate different regions of antioxidant activity and phenolic content yield based on different shades. It can be seen that potential antioxidant increases with increase in dextrose, yeast extract and incubation time. Low level of each of the factor reported low antioxidant activity.

However, incubation time seems to play a critical role in enhancing the antioxidant activity of the extract as can be seen in the contour plots. This might be due to the slow growth rate of the fungal strain. The maximum yield falls in the range $10-20 \mathrm{~g} / \mathrm{l}$ of dextrose $1-2 \mathrm{~g} / \mathrm{l}$ yeast extract and 20-25d for incubation time as indicated by dark shaded region in the plot. Hence to optimize the levels of dextrose, yeast extract and incubation time it is necessary to carry out trials in the region showing a high yield of antioxidants.
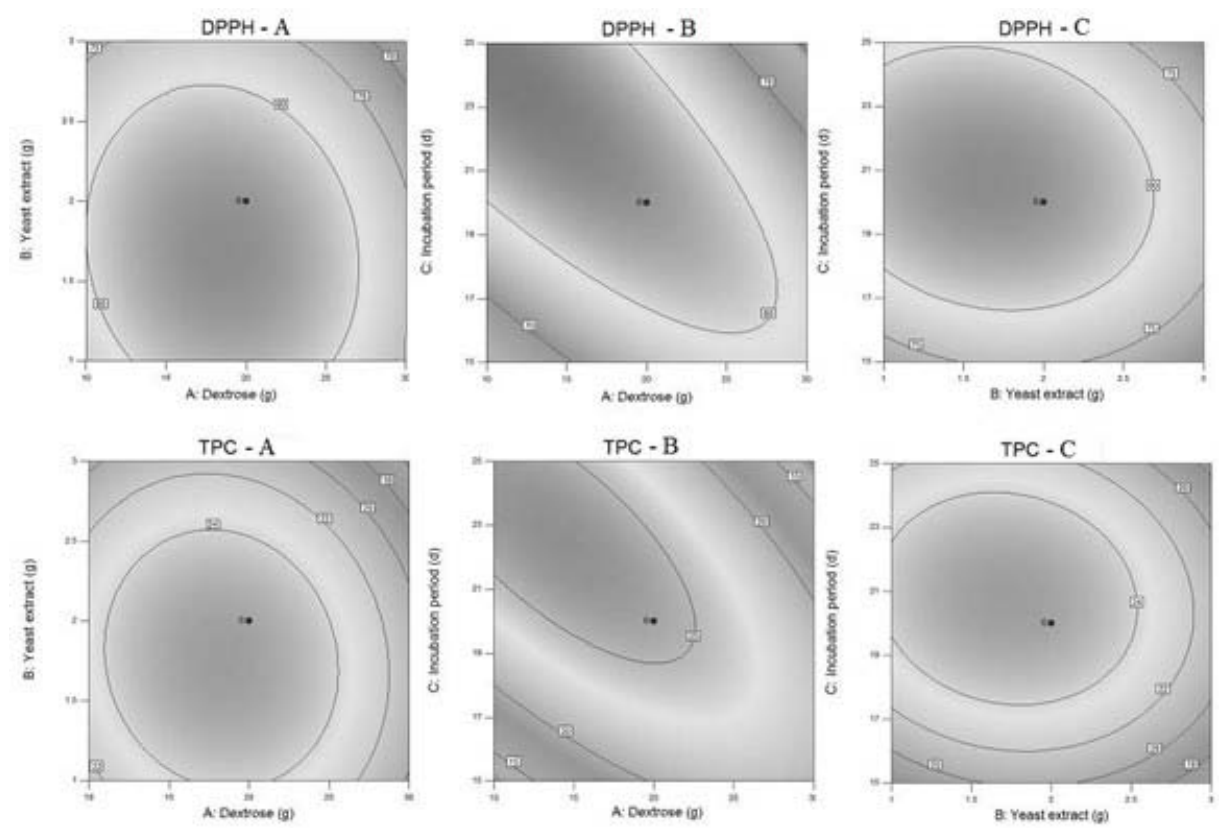

Fig. 1: Contour plot for interaction of different factors in CCD. DPPH: A, B, C: Contour plots of different factors for DPPH activity; TPC: A, B, C: Contour plots of different factors for total phenolic content 


\section{Antioxidant activity using optimized conditions}

From RSM, the optimized conditions for enhanced values for DPPH, NO and TPC may be concluded as, $12.731 \mathrm{~g}$ dextrose, $1.674 \mathrm{~g}$ yeast extract and $23.427 \mathrm{~d}$ of incubation time. To support that the predicted model has maximum antioxidant activity, an analysis was done using the optimum medium conditions. The trial under optimized conditions was carried out in triplicates. The ethyl acetate extract showed values

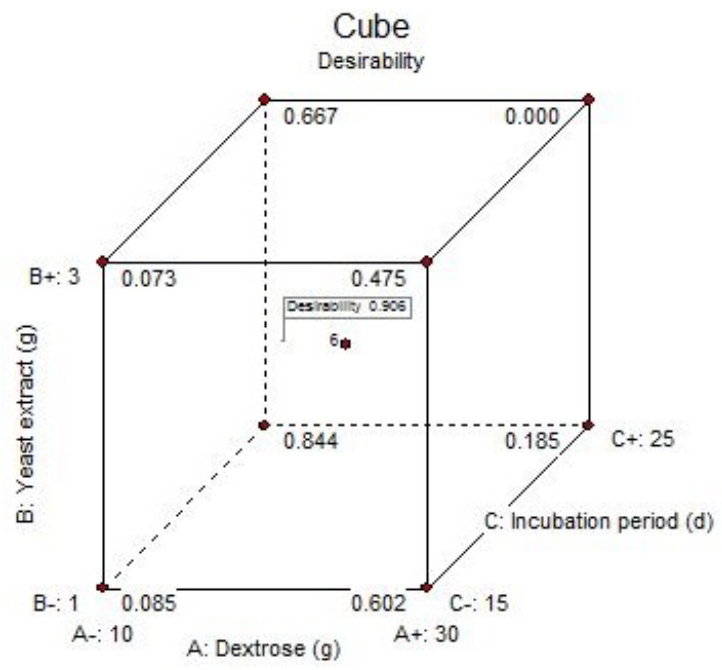

Cube

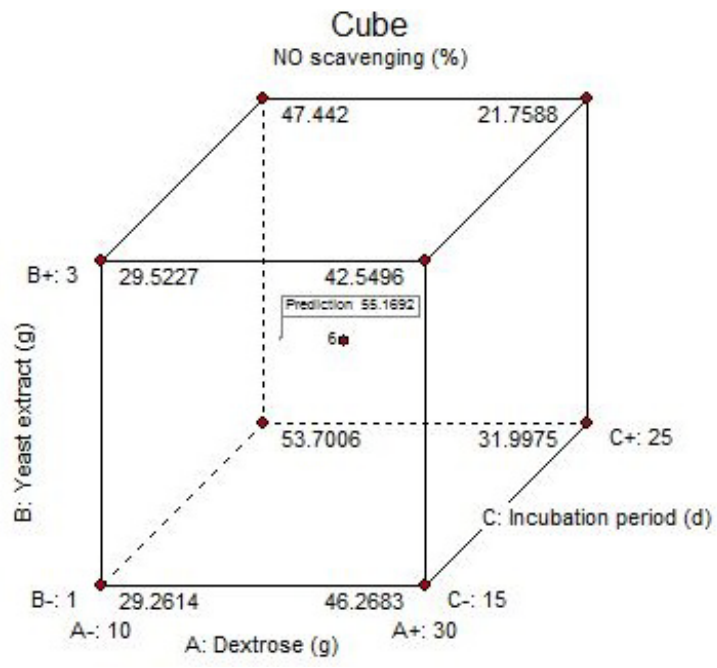

of $87.38 \%, 56.43 \%, 27.28 \mathrm{mg} / \mathrm{g}$ for DPPH radical scavenging, NO scavenging assay and total phenolic content respectively.

There is a strong agreement between the predicted and actual results (fig. 2) which authenticates the validity of the model. The enhancement of antioxidant potential of the extract indicates that RSM is an effective device to determine the optimal conditions and values for the required response.
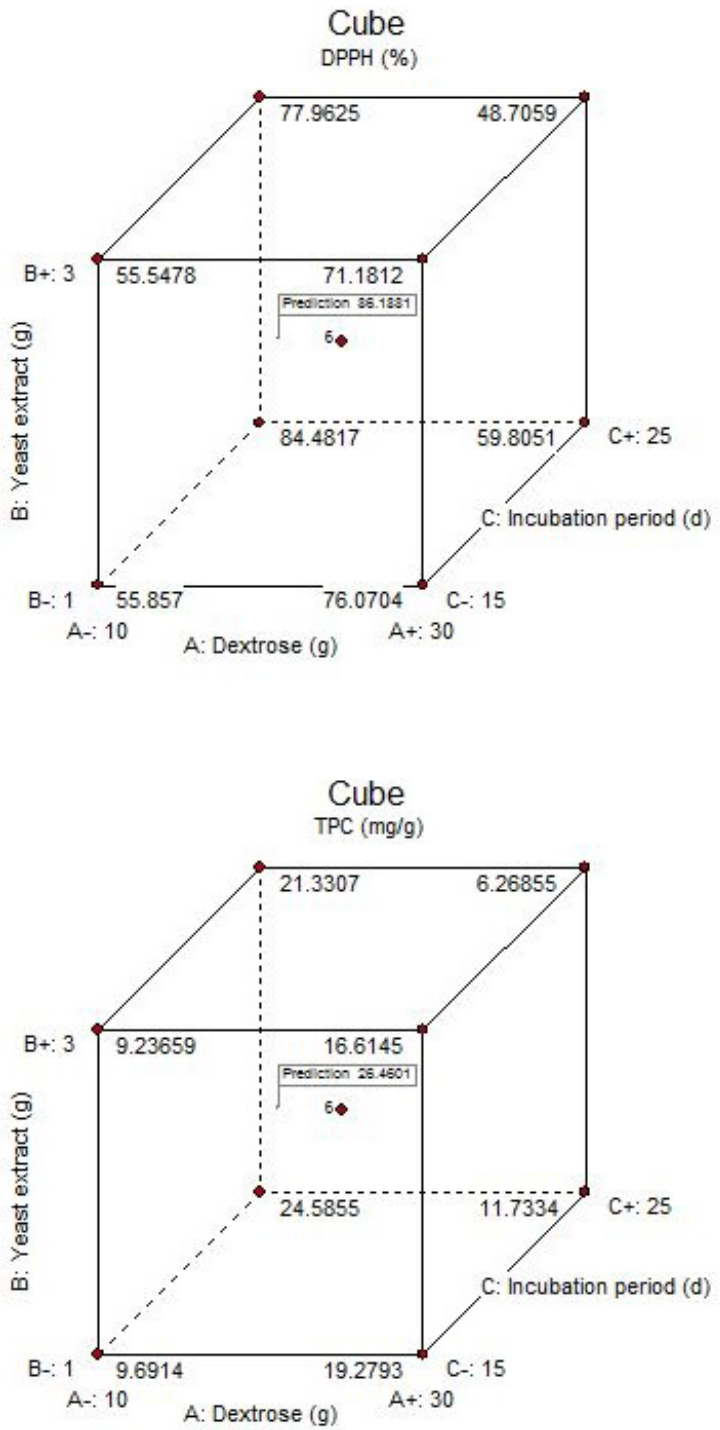

Fig. 2: Cube plot for optimization by CCD. Cube plot of A) Desirability B) Dextrose C) Yeast extract D) Incubation time

\section{DISCUSSION}

The design of culture media has a major role in the growth and the production of secondary metabolites [9]. Substantial work has been done on finding out the antioxidant activity of endophytic fungi, yet a lot has to be explored in improving the culture design for optimizing the antioxidant activity. The importance of carbon and nitrogen source in regulating secondary metabolite production has been reported many times [10]. Analysis of the effect of the medium constituents through Plackett-Burman design showed dextrose and yeast extract to be significant, but the significance of potato infusion was less $(\mathrm{P}>\mathrm{.05})$. Further, CCD (RSM) showed that yeast extract in the medium favors the production of metabolites having antioxidant activity. Dextrose is beneficial for the growth of fungi, and at high concentrations of dextrose, there was a substantial increase in growth of fungus and not its antioxidant activity. It may be assumed that at high concentrations of dextrose fungal species may prefer to utilize the carbon source for vegetative growth rather than the production of secondary metabolites. Though potato infusion did not significantly affect the antioxidant activity it is still retained at a standard concentration in PDYEB because components of the infusion are required by all the fungi for a variety of regulatory functions and control the biosynthesis of various secondary metabolites. The importance of incubation time in microbial growth and biosynthesis has been previously reported. In many cases maximum amount of secondary metabolites are produced when the fungal strain reaches a stationary phase in growth. About 20-25 d of incubation period was found the optimum for the antioxidant activity of the fungal strain, and the subsequent decline in bioactivity could be due to the exhaustion of nutrients available for the fungi or 
degradation of the secondary metabolites produced by the fungi. The large incubation period compared to other fungal species like Aspergillus and Penicillium can be due to the slow rate of growth of the strain. Earlier studies have shown that there is a linear correlation between the antioxidant activity and phenolic content [11]. These studies suggest that anti-oxidative effects of organisms are primarily due to redox properties of phenols which can play a significant role in neutralizing free radicals [12]. In the present study total phenolic content of $X$. feejeensis correlated effectively with antioxidant activity measured by DPPH and NO scavenging assay. The extract obtained from Xylaria feejeensis showed high activity against DPPH. It neutralized the purple color of DPPH to yellow color diphenyl picryl hydrazine molecule. This might be due to the hydrogen donating ability of the phenols present in the extract. The positive correlation between DPPH and TPC ( $r=0.992)$ supports this assumption. The present study also found that there is positive correlation between NO scavenging activity and phenolic content of the extract $(r=0.989)$. An increased production of phenolics under optimized conditions by P-B design and CCD further enhanced the antioxidant activity authenticating the relationship between antioxidant activity and phenolic content.

\section{CONCLUSION}

The study demonstrates the importance of statistical optimization techniques like P-B design and RSM in enhancing the antioxidant activity of the fungal strain Xylaria feejeensis HMJAU22039. Further research on bioactivities of the fungal extract can reveal the possibilities of any new findings that may have pharmacological significance.

\section{CONFLICTS OF INTERESTS}

\section{Declared none}

\section{REFERENCES}

1. Santhosh Goveas W, Asha Abraham. Evaluation of antimicrobial and antioxidant activity of stem and leaf extracts of Coscinium fenestratum. Asian J Pharm Clin Res 2013;6:218-21.

2. Pracheta, Veena Sharma, Ritu Paliwal, Sadhana Sharma. In vitro free radical scavenging and antioxidant potential of ethanolic extract of Euphorbia neriifolia Linn. Int J Pharm Pharm Sci 2011;3:238-42.

3. Robin Sharma, Rashmi R, Vijaya Kumar BS. Application of plackett-burman design to evaluate media components affecting antioxidant activity of an endophytic fungus Penicillium sp. isolated from the bark of Aegle marmelos. Int J Pharm Biol Sci 2014;5:1010-9.

4. Swati Hegde, Gururaj Bhadri, Kavita Narsapur, Shanta Koppal. Statistical optimization of medium components by response surface methodology for the enhanced production of bacterial cellulose by Gluconacetobacter persimmonis. J Bioprocess Biotech 2013;4:1.

5. Rahayu Ahmad, Najeeb Kaid Nasser Al-Shorgani, Aidil Abdul Hamid. Optimization of medium components using response surface methodology (RSM) for mycelium biomass and exopolysaccharide production by Lentinus squarrosulus. Adv Biosci Biotechnol 2013;4:1079-85.

6. Zhao, Xiang GR, Ye ZJ. Antioxidant activities of Salvia miltiorrhiza and Panax notoginseng. Food Chem 2006;99:767-74.

7. Kang KS, Yokozawa T, Kim HY. Study on the nitric oxide scavenging effects of Ginseng and its compound. J Agric Food Chem 2006;54:2558-62.

8. Singleton VL, Ortofehr R, Lamuela-Raventos RM. Analysis of total phenols and other oxidation substrate and antioxidants by means of folin-ciocalteau reagent. Methods Enzymol 1999;299:152-78.

9. Miao LI, Kwong TFN, Qian PY. Effect of culture conditions on mycelial growth, antibacterial activity, and metabolite profiles of the marine-derived fungus Arthrinium c. f. saccharicola. Appl Microbiol Biotechnol 2006;72:1063-73.

10. Gogoi DK, Deka Boruah HP, Saikia R, Bora TC. Optimization of process parameters for improved production of the bioactive metabolite by a novel endophytic fungus Fusarium sp. DF2 isolated from Taxus wallichiana of North East India. World J Microbiol Biotechnol 2008;24:79-87.

11. Zhao GR, Xiang ZJ, Ye TX, Yuan YJ, Guo ZX. Antioxidant activities of Salvia miltiorrhiza and Panax notoginseng. Food Chem 2006;99:767-74.

12. Huang WY, Cai YZ, Hyde KD, Corke H, Sun M. Endophytic fungi from Nerium oleander L (Apocynaceae): main constituents and antioxidant activity. World J Microbiol Biotechnol 2007;23:1253-63.

\section{How to cite this article}

- Durga Rebbapragada, Rajagopal Kalyanaraman. Statistical optimization of medium components by Plackett Burman design and response surface methodology for enhanced antioxidant activity by Xylaria feejeensis HMJAU22039. Int J Pharm Pharm Sci 2016;8(11):159-164. 